Introduction Outpatient visits to secondary care are costly to Primary Care (PC), time consuming for patients and not always appropriate. Our Trust having been a pioneer in nephrology eConsultation (eC), we started a hepatology eC service in March 2012. This allows patients' records to be shared between PC and secondary care (SC) without the need for a detailed referral letter.

Our aims were to analyse our initial experience and perform a cost analysis of hepatology eC.

Methods Retrospective analysis of eC between Mar 12- Oct 13 using SystmOne. Tariffs used for cost analysis: new patient (NP) referral $£ 181$, follow up (FU) $£ 103$, eC $£ 24$.

Results In 18 months there were $81 \mathrm{eC}(31 \mathrm{M} / 50 \mathrm{~F}$ mean age $52 /$ 56 y), each taking 10-15 min to complete. The median response time was 2 days (43\% within 1 day). Referral reasons: isolated raised bilirubin/ALT/ALP/GGT: 44/81 (54\%), mixed raised LFT's: $16 / 81$ (20\%), abnormal radiology 10\%, hyperferritinaemia 9\%, HBV/HCV 4\%, general advice 2\%. There was one inappropriate referral. In 18 cases, SC referral was recommended (22\%), with 10/18 being referred and seen. The mean number of FU appointments was 3. Total cost to PC was $£ 8,114$ : eC $£ 1,944$ and $£ 6,170$ for subsequent referrals. A minimum cost saving of $£ 14,890$ was made (81 NP $(£ 14,661)$ and assuming one $\mathrm{FU}$ for each $(£ 8,343))$.

Conclusion eC is a rapid, cost-effective method of providing hepatology advice. Hidden costs including consultant time, clinic costs etc are difficult to quantify. We would, however, recommend $\mathrm{eC}$ as the way forward with a more appropriate tariff. Disclosure of Interest None Declared.

\section{PTU-133 PREDICTING MORTALITY IN ALCHOLIC HEPATITIS; A COMPARISON IN DIFFERENT SCORING SYSTEMS}

SK Sidhu*, C Reddick, A Turner, K Mcwhirter, A Al-Rifai. Salford Royal Hospital, Manchester, UK

\subsection{6/gutjnl-2014-307263.207}

Introduction Alcoholic hepatitis is a severe presentation of alcoholic liver disease. In its most severe form (with a Maddray score $>32$ ) the 1 month mortality is $35 \%$. There are currently a few methods used to decide if continuation of corticosteroid therapy is beneficial, the Lille's Score and the presence of a 25\% reduction in the serum bilirubin between day 1 and day 7 . We assess the effectiveness of these scoring systems in assessing 6 month mortality.

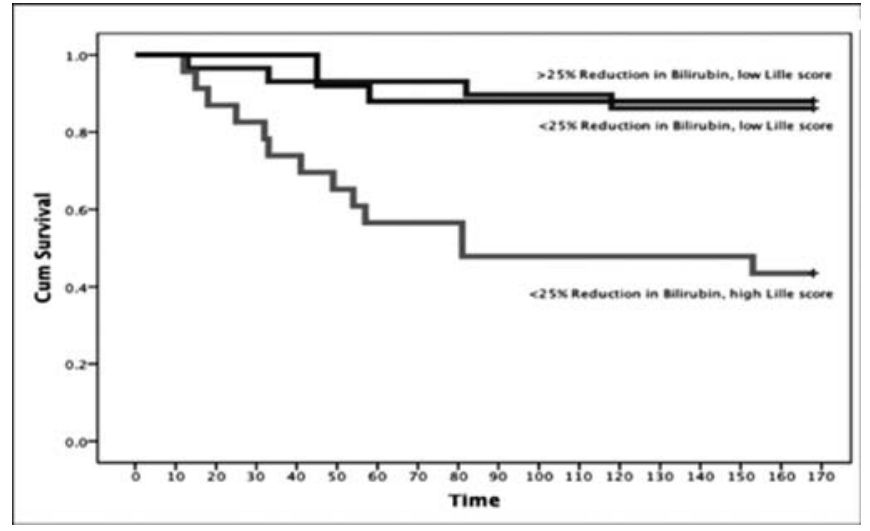

Abstract PTU-133 Figure 1 Kaplan-Meier survival curve at 6 months post admission
Methods All patients admitted with a diagnosis of alcoholic hepatitis with a Maddray score $>32$ with no evidence of sepsis over a 2 year period (2010-2012) were identified. All the notes were analysed and data collected by a F1 using a standardised profroma. Patients were placed into 4 groups: Group $1=<25 \%$ reduction in bilirubin, low Lille score, Group $2=<25 \%$ in bilirubin, high Lille score, Group $3=>25 \%$ reduction in bilirubin, low Lille score and Group $4=>25 \%$ reduction in bilirubin, high Lille score.

Results Overall 77 patients were included, at 6 months 21 died (27\%). The mean age was 48 yrs (Range 27-67 years). At day 1 there was biochemical parameters consistent with significant liver disease (Madray Score: 68 (Range 34-169), Albumin $29.9 \pm$ 4.9, INR: $2.0 \pm 0.5$, Bilirubin: $235 \pm 135) .77$ (100\%) patients received nutritional support, Vitamin $\mathrm{B}$ and Thiamine. The baseline INR (Alive: $1.85 \pm 0.47$, Died: $2.31 \pm 0.59 \mathrm{p}=0.001$ ) and Albumin (Alive: $30.6 \pm 4.6$, Died: $28.1 \pm 5.5 \mathrm{p}=0.04$ ) were significantly deranged in patients who died at 6 months. There was no significant difference in the baseline Urea (Alive: $3.81 \pm 3.54$, Died: $4.26 \pm 3.46 \mathrm{p}=0.610$ ), Creatinine (Alive: $64.3 \pm 45.9$, Died: $79.5 \pm 59.6 \mathrm{p}=0.232)$ and Bilirubin (Alive: $237.8 \pm 144.6$, Died: $228.1 \pm 110.3 \mathrm{p}=0.779$ ) in patients who died at 6 months.

There were no patient that fell into group 4 . In the other 3 groups there were similar numbers of patients (Group 1: 29 patients, 6 month mortality 17\%, Group 2: 23 patients, 6 month mortality 57\%, Group 3: 25 patients, 6 month mortality 12\%). Kaplan Meier survival curves were created for these 3 groups and is shown in Figure 1 below.

Conclusion In this study factors suggesting poor liver synthetic function (INR and Albumin) were associated with 6 month mortality. There was a significantly worse outcome with a high Lille score compared to a low Lille score. There was very little effect of a greater than $25 \%$ reduction in bilirubin on mortality at 6 months. From this study we would suggest that the Lille score is used to accurately predict a poor outcome.

Disclosure of Interest None Declared.

\section{PTU-134 DURHAM PATHWAY FOR CARE OF PATIENTS WITH ADVANCE STAGE LIVER DISEASE (ASLD)}

${ }^{1} S$ Saksena*, ${ }^{2} \mathrm{~L}$ Hammal, ${ }^{2} \mathrm{M}$ Hewett, ${ }^{2} \mathrm{C}$ Lancaster. ${ }^{1}$ Hepatology, Durham, UK; ${ }^{2}$ County Durham Darlington Foundation Trust, Durham, UK

\subsection{6/gutjnl-2014-307263.208}

Introduction Care of patients with ASLD is poorly organised. This service improvement project aimed to develop a consistent pathway of care for ASLD patients.

Methods Methods and results were iterative and included: 1 . Track patient journey and Carer Experience: 2. Capture Activity Data from Clinical Coding: 3. Process mapping patient journey and Identify gaps in service provision 4. Design "Durham pathway" Figure for improving care of patients with ASLD with in reach liver service in community and developed range of both quantitative and qualitative metrics "Durham metrics" to monitor the effectiveness of the new pathway on patient outcomes and experience. 5. Engage relevant stakeholders and shared pathway 6. A 6 month Pilot of 20 ASLD patients with Community matron led inreach service. 7 . Engage commissioners to fund ASLD pathway.

Results Pre-pilot patient and carer experience was poor with multiple unplanned admissions, preferred place of death was not discussed, majority of deaths in hospital, care was not 


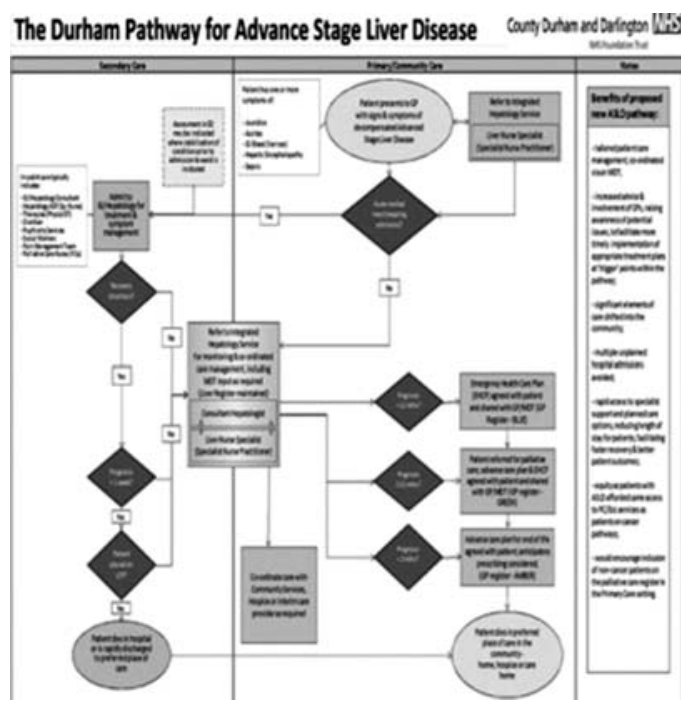

Abstract PTU-134 Figure 1

coordinated, and quality of life of patient and carer was poor. All post-pilot metrics reported significant improvements. Improved efficiency evidenced by reduction in unplanned hospital admissions, increase use of alternative community services, with use of shared care plans. $83 \%$ of these achieved their preferred place of care and death in contrast to nil pre pilot. Conclusion This pathway worked for ASLD and needs wider evaluation and consideration of similar approaches to other groups. Disclosure of Interest None Declared.

\section{PTU-135 ELAAR (THE ENHANCED LIVERPOOL ABDOMINAL AREA RATIO): HOW TO USE CROSS-SECTIONAL IMAGING TO ASSESS PROGNOSIS IN END-STAGE CIRRHOSIS}

${ }^{1}$ SM Alam*, ${ }^{2} \mathrm{C}$ Farrell, ${ }^{3}$ I Patanwala, ${ }^{3} \mathrm{P}$ Richardson, ${ }^{3} \mathrm{~T}$ Cross. ${ }^{1}$ Gastroenterology, Royal Liverpool and Broadgreen University Hospital Liverpool UK; ${ }^{2}$ Radiology, Royal Liverpool University Hospital, Liverpool, UK; ${ }^{3}$ Gastroenterology, Royal Liverpool University Hospital, Liverpool, UK

\subsection{6/gutjpl-2014-307263.209}

Introduction Liver size may be important in prognostication in cirrhosis. The LAAR score has previously shown a relationship between liver size and survival but is hampered by subjectivity. The current study aimed to improve the existing score.

Methods A retrospective-prospective cohort study was performed on patients with cirrhosis. The censor point used was date of patient death or liver transplant (LT) from CT date. Time points were measured from the date of the CT scan to censor point or last clinic appointment. The enhanced Liver ${ }^{\text {pool }}$ to Abdomen Area Ratio (eLAAR) was derived using a software package (Carestream). eLAAR was calculated using the formula (Liver area $\left(\mathrm{cm}^{2}\right)$ /Abdominal area $\left(\mathrm{cm}^{2}\right) \times 100$.

Results 101 patients were identified, 66\% male, median age 52 (44-60 years). The LAAR score detected progression to death/ LT in our cohort at 1 year $(\mathrm{p}=0.02)$ and at 5 years $(\mathrm{p}=0.03)$. The intra-class correlation coefficient between 2 operators was 0.94 (95\% CI 0.89-0.97). Using an optimal eLAAR cut-off of 32 eLAAR could predict death at 1 and 5 years from diagnosis, $\mathrm{p}=0.03($ OR 2.51(1.08-2.51) and $\mathrm{p}=0.002() \mathrm{R} 3.9895 \%$ CI 1.5-10.4). Survival curves were constructed and the log rank test showed that eLAAR was able to predict death at 1 year ( $\log$ rank 5.3, $\mathrm{p}=0.02)$ and 5 years (log-rank, $\mathrm{p}=9.7, \mathrm{p}=0.002)$.
Conclusion The eLAAR score offers a new paradigm to identify patients with poor prognostic criteria on cross-sectional imaging who may benefit from liver transplantation.

Disclosure of Interest None Declared.

\section{PTU-136 DOES A NORMAL IGG INDICATE HISTOLOGICAL REMISSION IN AUTOIMMUNE HEPATITIS (AIH)?}

'VM Gordon*, 'E McFarlane, ${ }^{2} \mathrm{~A}$ Dube, ${ }^{1} \mathrm{D}$ Gleeson. ${ }^{1}$ Hepatology, Sheffield Teaching Hospitals NHS Trust, Sheffield, UK; ${ }^{2}$ Histopathology, Sheffield Teaching Hospitals NHS Trust, Sheffield, UK

\subsection{6/gutjnl-2014-307263.210}

Introduction Response to immunosuppressive treatment in $\mathrm{AIH}$ is often monitored by measurement of serum immunoglobulin $G$ (IgG) as well as ALT. It is commonly assumed that serum IgG level correlates with histological activity (or Ishak necroinflammatory score: NIS) on liver biopsy, the historical "gold standard". However, only one group (Luth et al 2008; J of Clin 42 (8):926-930.) have examined this relationship, finding that normalisation of both serum ALT and IgG reliably predicted a NIS of $<6$ but not a NIS of $<4$ (corresponding to minimal hepatitis, seen in less than half of the patients presumed to be in remission). Here, we aimed to reassess how well serum $\operatorname{IgG}$ correlated with NIS in treated patients with AIH undergoing follow-up biopsy for confirmation of disease remission.

Methods We assessed 31 follow-up biopsies, performed to confirm histological remission in 28 patients with AIH (International Group criteria; Alvarez J Hepatol 1999; 31:929) on immunosuppressive treatment and an accompanying serum IgG (measured within 6 weeks of biopsy).

Results For 29 of the 31 follow-up biopsies, accompanying serum IgG was in the normal range ( $\leq 16 \mathrm{gm} / \mathrm{L})$. However, only 13 of these 31 biopsies showed NIS $<4$ (minimal hepatitis). On ROC analysis, area under the curve (AUC) for IgG in predicting a NIS of $\geq 4(\mathrm{n}=31)$ was $0.596(\mathrm{p}=0.368)$. Sensitivity and specificity of IgG (cut off $>16 \mathrm{grm} / \mathrm{L}$ ) in predicting a NIS of $\geq 4$ was $5.5 \%$ and $57 \%$ respectively. Corresponding positive (PPV) and negative (NPV) predictive values were only 50\% and 59\% respectively. AUC for change in $\operatorname{IgG}(\triangle \mathrm{IgG}$ : baseline values minus values accompanying follow up biopsy; $\mathrm{n}=29$ ) in predicting NIS $>4$ was $0.551(\mathrm{p}=0.642)$.

Defining histological remission instead as minimal or mild hepatitis- NIS < 6 (as Luth's group did because they found that such patients did not develop fibrosis progression), there were still 4/29 (14\%) patients with normal serum IgG who were not in histological remission. On ROC analysis, AUC for IgG in prediction of NIS $>6$ on follow-up biopsy $(\mathrm{n}=31)$ was $0.62(\mathrm{p}=$ 0.39 ). PPV and NPV for serum IgG (cut off $>16 \mathrm{grm} / \mathrm{L}$ ) in predicting of NIS $\geq 6$ ) was $50 \%$ and $86 \%$ respectively. AUC for $\Delta \mathrm{IgG}$ in prediction of NIS $\geq 6$ was 0.608 ( $\mathrm{p}=0.453$ ).

Conclusion Normalisation of serum IgG values in patients with $\mathrm{AIH}$ following treatment is not predicative of histological remission. At present, this requires a liver biopsy.

Disclosure of Interest None Declared.

\section{PTU-137 PNEUMOCOCCAL VACCINATION IN PATIENTS WITH LIVER CIRRHOSIS - IS THE MESSAGE BEING HEARD?}

V Sehgal* ${ }^{*}$ H Alexander, K Besherdas. Gastroenterology, Chase Farm Hospital, London, UK

10.1136/gutjnl-2014-307263.211 